\title{
Predictor of Atrial Fibrillation Recurrence in Patients who Underwent a Tricuspid Valve Operation with Modified Cox Maze procedure
}

\section{Minjung Bak}

Samsung Medical Center

Sung-Ji Park ( $\nabla$ tyche.park@gmail.com )

Samsung Medical Center https://orcid.org/0000-0002-7075-847X

\section{Dong Seop Jeong}

Samsung Medical Center

\section{Boram Park}

Samsung Medical Center

Jeong Hun Seo

Kangwon National University Hospital

Ilkun Park

Samsung Medical Center

Jihoon Kim

Samsung Medical Center

\section{Su Ryeun Chung}

Samsung Medical Center

Eun Kyoung Kim

Samsung Medical Center

Kiick Sung

Samsung Medical Center

\section{Research}

Keywords: tricuspid valve operation, maze, right atrium diameter, atrial fibrillation, structural heart disease

Posted Date: May 18th, 2021

DOI: https://doi.org/10.21203/rs.3.rs-533155/v1

License: (1) (1) This work is licensed under a Creative Commons Attribution 4.0 International License.

Read Full License 
Version of Record: A version of this preprint was published at Echocardiography on February 14th, 2022. See the published version at https://doi.org/10.1111/echo.15315. 


\section{Abstract}

Background Recurrence of any atrial arrhythmia after surgical ablation is known as a negative predictor of cardiovascular events and total mortality. However, there have been no focused studies for atrial fibrillation (AF) recurrence prediction in patients with significant tricuspid regurgitation (TR), and the riskbenefit estimation of surgical ablation in tricuspid valve (TV) surgery is not fully established.

Objectives In this study, we analyzed predictors of AF recurrence, safety, and efficacy of the modified Cox maze (CM) procedure in patients with AF undergoing TV operation.

Method We screened 421 patients who underwent a TV operation between 1994 and 2017. After excluding patients who did not undergo a maze operation, 158 patients were enrolled. Enrolled patients were divided by recurrence of AF.

We analyzed the difference between the AF recurrence group and no AF recurrence group, and AF recurrence factors in terms of clinical risk factors and echocardiographic risk factors. The hazard ratio (HR) and 95\% confidence intervals (Cls) were presented using a Cox proportional hazard model.

ResultS Among 158 patients, AF recurred in 65 patients within 10 years. For AF prediction, age was most the important clinical factor and right atrium (RA) diameter was the most important echocardiographic parameters. In patients with a larger RA diameter over $49.2 \mathrm{~mm}$, the prevalence of AF recurrence was higher (HR 4.322, 95\% Cl [2.185-8.549], log rank $p$ value < 0.001).

In clinical outcome, there was no significant difference between the AF recurrence group and the no recurrence group in terms of death, TR recurrence, heart failure and stroke. However, the risk of permanent pacemaker (PPM) insertion was higher in the AF recurrence group (HR 4.706, 95\% Cl [0.975-22.708], log rank $\mathrm{p}$ value 0.034 ) compared to the no recurrence group.

Conclusion Age and RA enlargement are key predictors of AF recurrence after TV operation with the CM procedure in patients with significant TR.

\section{Introduction}

Ablation of atrial fibrillation (AF) can reduce the risk of cardiovascular events compared to medical treatment, and recurrence of any atrial arrhythmia after ablation can be a predictor of cardiovascular events and total mortality ${ }^{1}$. The benefits of surgical ablation in structural heart disease (SHD are well recognized ${ }^{2}$, and more than half of the patients with AF undergoing open-heart surgery have concomitant AF surgery these days ${ }^{3}$. Despite ablation, the risk of AF recurrence still remains, and AF recurrence can have negative effects on survival, heart failure exacerbation, and stroke risk ${ }^{4}$.

AF recurrence risk estimation after ablation is not fully established yet. In catheter ablation, investigation of $\mathrm{AF}$ recurrence prediction is active due to high numbers of interventions and anticoagulation issues. 
However, predictors of AF recurrence after surgical ablation are not as established as those of catheter ablation, and analysis of AF recurrence is usually conducted in patients who undergo general cardiac

surgery or mitral valve surgery ${ }^{5,6}$. In particular, there is no concerted study for patients with significant tricuspid valve (TV) disease.

The Maze operation is controversial in patients with significant tricuspid regurgitation (TR) resulting from SHD due to concern of that AF may recur regardless of whether or not the Maze operation is performed. There is no established risk benefit estimation for Maze operation in TV surgery. Therefore, in this study, we analyzed predictors of AF recurrence after a modified Cox Maze (CM) procedure in patients with AF who underwent a TR operation and attempted to identify clear cutoffs for the benefits and safety of the CM procedure in TV surgery.

\section{Methods}

\section{Study subject and data collection.}

We retrospectively investigated 421 patients who underwent a TV operation between 1994 and 2017 at Samsung Medical Center in Seoul, South Korea. We enrolled 158 patients who had significant TR with AF who underwent TV repair or replacement and CM procedure. Patients who did not have the CM procedure, did not have a follow-up ECG after three months post-op, or had inadequate echocardiographic measurements were not included. Patients were divided by recurrence of AF within 10 years after the TV operation with CM procedure (Figure 1).

The primary endpoint was AF recurrence. We analyzed the difference between the AF recurrence group vs. no recurrence groups, and analyzed the AF recurrence factor in terms of clinical and echocardiographic risk factors. Secondary end points were clinical outcomes in the two groups, which included death, TR recurrence, heart failure (HF) admission, permanent pacemaker (PPM) insertion and stroke events. The median clinical outcome follow-up duration was 7.9 years.

The medical records of the enrolled patients were carefully reviewed by research coordinators. Mortality data for patients who were lost to follow-up were confirmed by National Death Records. The study protocol was approved and the requirement for informed consent of the individual patients was waived by the Institutional Review Board of Samsung Medical Center. This study was conducted according to the principles of the Declaration of Helsinki. (IRB No. 2020-12-054)

\section{Surgical technique.}

Detailed techniques of the cryo-maze procedure were described in our previous report ${ }^{7}$. The cryo-maze procedure was performed with an N2O-based cryoprobe or an argon-based cryoprobe according to surgeons' preference. 
Usually, five lesions were created, including pulmonary vein isolation, mitral isthmus, posterior part of left atrium extending to the left atrial appendage to box the lesion, cavo-tricuspid-isthmus, and superior vena cava to inferior vena cava line. Ablation time was 180 seconds. After completion of this procedure, additional cardiac procedures including valve surgery, CABG, or ASD closures were performed. The opening left atrial appendage was internally obliterated without an excision using a running 3-0 monofilament suture.

\section{Definitions}

$A F$ recurrence was defined as restoration of $A F$ rhythm at least one time in follow up electrocardiography (ECG) more than three months after the operation. Patients who never returned to sinus rhythm were counted in the AF recurrence group and recurrence day was set as zero.

Structural heart disease (SHD) in this study was defined as more than a moderate degree of valve disease, previous cardiac operation history, or congenital heart such as like atrial septal defects (ASD). Isolated TR without SHD indicates secondary TR caused by atrial fibrillation. Significant VHD indicates more than a moderate degree of valve disease.

Chronic kidney disease was defined as a glomerular filtration rate (GFR) $<60 \mathrm{~mL} / \mathrm{min} / 1.73 \mathrm{~m}^{2}$ over 3 months and a disease code in medical record. Coronary artery disease was defined as over $50 \%$ stenosis in at least one coronary artery on computed tomography (CT) angiography or coronary angiography. TR recurrence was defined as reappearance of more than a moderate degree of TR after restoration to a minimal or mild degree at early after surgery. Stroke was defined as a neurological deficit of abrupt onset caused by ischemia or hemorrhage within the brain.

\section{Echocardiographic evaluation.}

Two-dimensional echocardiography was performed using commercially available equipment (Vivid 7, GE Medical Systems, Milwaukee, Wl; Acuson 512, Siemens Medical Solution, Mountain View, CA; or Sonos 5500, Philips Medical System, Andover, MA). End diastole was defined as the frame with the largest cavity area immediately before the onset of the QRS and end systole as the frame with the smallest cavity area. At rest, left ventricular end-diastolic dimension (LVEDD) and LV end-systolic dimension (LVESD) were obtained from parasternal views according to the American Society of Echocardiography (ASE) guidelines ${ }^{8}$. LV ejection fraction (EF) were calculated from two-dimensional recordings using the modified biplane Simpson's method. Relative wall thickness (RWT) and left ventricular mass (LVM) were calculated from linear dimensions using the ASE-recommended formula. Left atrial (LA) volume was assessed by the modified biplane area-length method and was indexed to body surface area. Early diastolic mitral inflow velocity $(E)$ was measured using the pulsed wave Doppler method by placing the sample volume at the level of the mitral valve leaflet tips. Tissue Doppler-derived early diastolic mitral annular velocity (e') was measured from the septal corner of the mitral annulus in the apical fourchamber view. We calculated the E/e' ratio as an index of left ventricular filling pressure. LA diameter was measured in the apical 4 chamber view or parasternal long axis view at end systole phase and LA 
diameter over $40 \mathrm{~mm}$ was defined as LA enlargement (LAE). RA diameter was measure in a dedicated right heart view from an apical 4 chamber view that includes the entire RA and was not fore-shortened at the end ventricular systole phase from the inner edge to the inner edge at the mid-atrial level. Right ventricular (RV) diameter, Tricuspid annular plane systolic excursion (TAPSE), and TDI-derived tricuspid lateral annular systolic velocity (TV s') were also measured in a dedicated right heart view. A RA diameter over $45 \mathrm{~mm}$ was defined as RA enlargement (RAE). Right ventricular systolic pressure (RVSP) was obtained by assumed RA pressure plus 4 * (maximal TR velocity) ${ }^{2}$. RA pressure were assumed by Inferior vena cava (IVC) diameter and the presence of plethora. IVC diameter was measured at the junction of the hepatic vein approximately $3 \mathrm{~cm}$ from the RA in a standard subcostal view at end expiration, perpendicular to the IVC long axis. Quantitative and qualitative measurements of TR severity were performed according to American Society of Echocardiography guidelines.

\section{Statistical analysis.}

Continuous variables are presented as mean \pm standard deviation or median [interquartile range (IQR)]. Differences between the $\mathrm{AF}$ recurrence group and no $\mathrm{AF}$ recurrence group were evaluated using student ttest or Mann Whitney $\mathrm{U}$ test. Categorical variables were compared between groups using the Chi-square test or Fisher's exact test and are presented as numbers and relative frequencies (\%).

Clinical factors were considered for old age (age over 60), Sex, DM, HTN, CKD, Stroke Hx, CAD, previous cardiac operation history, and TV operation method (TV repair or replacement). We also examined echo parameters including LVEF, LAVI, RAD, LVEDD, RVSP, E/e', TR grade, and ASD closure. The univariable and multivariable Cox proportional hazard model was applied to estimate the hazard ratio (HR) and 95\% confidence interval $(\mathrm{Cl})$ for AF recurrence. The survival curves were extracted using the Kaplan-Meier method. Variance inflation factors (VIF) of clinical factors ranged from 1.055 to 1.374 . The VIFs of echo parameters ranged from 1.069 to 1.725 . For clinical factors and echo parameters, the final multivariable regression model was determined by backward variable selection method with criteria of $p$-value $<0.05$. The $\mathrm{C}$ index or concordance $\mathrm{C}$ was considered an overall measure of discrimination in survival analysis, and we tested whether there was a difference between two correlated overall $C$ indices. Cut-off values were determined by the slope and intercept value of a generalized linear model.

All p-values were two-sided, and p-values $<0.05$ were considered statistically significant. Statistical analyses were performed using R Statistical Software (version 3.6.0; R Foundation for Statistical Computing, Vienna, Austria) and SPSS statistics 20 (SPSS Inc., Chicago, IL).

\section{Results}

\section{PredictorS of AF recurrence.}

Among 158 patients who underwent TVR or TV repair with CM procedure, 136 patients (86.1\%) of them had SHD and the remainder ( $n=22)$ had isolated TR without SHD. 134 patients (84.8\%) of them had a TR repair and the remaining 24 patients (15.2\%) had a TV replacement. 11 (7.0\%) patients had an ASD 
closure and $8(5.1 \%)$ patients had a concomitant CABG. 65 of 158 patients $(41.1 \%)$ had recurrent AF within 10 years while 93 patients (58.9\%) maintained sinus rhythm without evidence of AF recurrence on follow up ECG.

In baseline characteristics analysis, the AF recurrence group were older (age over 60) and had a larger RAD. There was no significant difference in sex, DM, HTN, CKD, stroke history, coronary artery disease, previous cardiac operation history, concomitant procedure, or surgical information between the AF recurrence group and the no recurrence group. With respect to echocardiographic parameters, there were no significant differences between the AF recurrence group and the no recurrence group except for RAD. LAD, LAVI, and LVEF were not significantly different between two groups. In addition, the proportion of patients with significant valve disease such as more than a moderate degree of mitral stenosis (MS), mitral regurgitation (MR), aortic stenosis (AS), aortic regurgitation (AR), and isolated TR were also not significant different between the two groups (Table 1).

In a multivariable Cox regression model among clinical factors (Table 2-1), old age was the only significant risk factor for AF recurrence. Among echocardiographic parameters, LAVI, RAD, and RVSP were important risk factors for $A F$ recurrence in the multivariable model (Table 2-2). The C-index value for AF recurrence within 10 years of old age (defined as age over 60 ) was $57.9 \%$. When we combined the prediction value of old age and echocardiographic parameters, which were both significant in multivariable Cox regression, only RAD had an additive value to the $\mathrm{C}$ index, at $67.7 \%$ ( $p$ value $=0.003$, when compared to the C-index of old age alone, which was 57.9\%). (Figure 2 )

The AUC value of solitary RAD was $66.5 \%$. The cut-off value for RAD was $49.2 \mathrm{~mm}$, and AF recurred more than 4 times in patients with RAD over 49.2mm (HR 4.322, 95\% Cl [2.185-8.549], p value < 0.001). (Figure 3) When comparing the AUC value of solitary echocardiographic parameters for AF recurrence prediction, RAD showed superior predictive value over LAVI. ( $p$ value $=0.036$, Supplemental Figure 1$)$

Comparing right heart-related echocardiographic parameters between the AF recurrence group and the no AF recurrence group, only IVC diameter showed a significant difference. The IVC diameter was higher in the AF recurrence group, although the RV dysfunction incidence estimated by TAPSE and TV s' did not differ significantly. Differences of RV size and TV annulus size were also insignificant. (Table 3)

In subgroup analysis with left-sided valve disease, the group with more than a moderate degree of MS showed unique baseline characteristics and significance of hazard ratio for AF recurrence according to RAD diameter. (Supplemental Table 1,2). In the MS group, the baseline RA diameter was not significantly different between the two groups (no AF recurrence group $50.9 \pm 7.3 \mathrm{~mm}$ vs. AF recurrence group $54.1 \pm$ $6.0 \mathrm{~mm}, \mathrm{p}$ value $=0.077$ ). However, LAVI did show a significant difference (no AF recurrence group 103.8 $\pm 44.0 \mathrm{~mm}$ vs AF recurrence group $137.9 \pm 66.6 \mathrm{~mm}$, $p$ value $=0.036$ ) in this subgroup, although no other group showed a significant difference in LAVI between the AF recurrence and no recurrence groups. RA diameter was not predictor for AF recurrence (HR 1.052, 95\% Cl [0.998-1.109], p value $<0.061$ ) in the MS group, even though all other subgroups showed significance in AF prediction according to RA diameter. In the MS group, LAVI seems more important than RA diameter. 
In subgroup analysis of SHD $(n=136,86.1 \%)$ and isolated TR $(n=22,13.9 \%)$, although RAD could predict AF recurrence in SHD (HR 1.052, 95\% Cl [1.028-1.076], $p$ value < 0.001), RAD did not correlate with AF recurrence in the isolated TR group (HR 1.009, 95\% $\mathrm{Cl}$ [0.959-1.062], $\mathrm{p}$ value < 0.724$)$. The interaction $\mathrm{p}$ value of RA diameter and the presence of isolated TR for AF recurrence was insignificant at 0.098 . The RA diameter of the AF recurrence group $(65.5 \pm 14.1 \mathrm{~mm})$ was numerically higher than that of the no recurrence group $(60.8 \pm 8.8 \mathrm{~mm})$ in patients with isolated TR, but there was no significant difference $(p$ value $=0.353)$.

\section{Clinical outcome.}

Long term, TR recurrence, $\mathrm{HF}$ admission, ischemic stroke, and hemorrhagic stroke showed no correlation with AF recurrence. Instead, the possibility of PPM insertion within the same period happened more frequently in the AF recurrence group. In patients who had recurrent AF within 10 years, 7 patients (15.1\%) had a PPM insertion, while only 2 patients (3.5\%) who maintained sinus rhythm needed PPM insertion. In the Cox proportional hazard model, the hazard ratio for PPM insertion in the AF recurrence group was $4.706(95 \% \mathrm{Cl}[0.975-22.708]$, log rank $\mathrm{p}$ value $=0.034)($ Table 4, Figure 4).

\section{Discussion}

In this study, we investigated predictors of AF recurrence and the safety and efficacy of a modified Cox maze procedure in patients with AF who underwent a TV operation. The major findings of this study were as follows: (1) RA enlargement is key predictor of AF recurrence after a TV operation with CM procedure in patients with significant TR; (2) AF recurred more than 4 times more commonly in patients with RAD over $49.2 \mathrm{~mm}$; and (3) risk of PPM insertion was higher in the AF recurrence group.

\section{Predictors of AF recurrence}

AF recurrence analysis based on echocardiographic parameters has been well-studied recently. Risk models like the APPLE score ${ }^{9}$ and MB-LATER score ${ }^{10}$ consist of clinical risk factors and echocardiographic parameters, and show superiority over the CHADS ${ }^{2}$ and $\mathrm{CHA}^{2} \mathrm{DS}^{2}$-VASc scores. These new risk prediction models use LA diameter as a scoring factor. LA diameter, LAVI, and LA strain ${ }^{11-14}$ are well known for $A F$ recurrence prediction in both lone AF and AF in SHD. This is also in line with the fact that pulmonary vein isolation is the most recommend strategy in $A F{ }^{15,16}$. Beside $L A V I, R A$ area ${ }^{13}, A F$ duration ${ }^{13,17}$ and size of $F$ wave ${ }^{17}$ are known for predicting AF recurrence. In this study, suppression of $A F$ recurrence after the $C M$ procedure with $T V$ surgery was up to baseline RAD and age. The relationship between RAD in AF occurrence was observed in mitral valve surgery ${ }^{13}$, catheter ablation ${ }^{18}$ and hypertrophic cardiomyopathy ${ }^{19}$. However, there are few studies that directly compare RAD with other echocardiographic parameters like LAD, LAVI or RVSP. In this study, LAVI had less discriminative value than RAD. $81 \%$ patients had left-sided valve disease and the average LAVI was $107 \mathrm{ml} / \mathrm{m}^{2}$, which is 3 times higher than the upper limit of normal. This suggests that there was no definite discriminative value in LAVI since LA dilatation had already progressed. 


\section{Safety and Efficacy of modified Cox Maze procedure in TR operation}

The pathophysiology of AF is reentry and atrial ectopic activity. Ablation for arrhythmia targets rhythm control by isolation of focal atrial ectopic activity. Concomitant surgical ablation of AF is recommended with level of evidence grade I in open surgical ablation and level II evidence in closed surgical ablation ${ }^{16}$. However, the beneficial effect of the maze procedure in TV surgery has not been discussed. In this study, the success rate of the CM procedure in TV surgery was $58.9 \%$ (93/158) without mortality. The cut-off value of RAD for AF recurrence was $49.3 \mathrm{~mm}$ and the PPM insertion rate was higher in the AF recurrence group. When deciding whether to perform a $\mathrm{CM}$ procedure in patients with more than a moderate degree of TR and AF, RAD could be an important factor for decision making based on this study's result.

Progression of TR is related to bi-atrial enlargement and tricuspid annulus dilatation ${ }^{20-22}$. In this study, most patients with moderate TR have RA enlargement (96.8\%) and RAD was the most important echocardiographic parameter for predicting AF recurrence. LA enlargement was also present in $96.8 \%$ of patients. The difference in IVC tended to be associated with RAD. The AF recurrence group had a larger IVC diameter, which could be explained by assumption that elevated RA pressure due to TR jet flow contributes to RA enlargement and IVC dilatation ${ }^{23}$ at the same time. RA enlargement made by a significant TR jet could lead to right atrial pathophysiological damage, and this change could contribute to $\mathrm{AF}$ recurrence after ablation.

Atrial enlargement is a feature in patients with SHD and in senescence. It is known that atrial myocardial structure relating to sinus node dysfunction (SND) is primarily in the right atrium ${ }^{24}$. This finding is in line with the fact that $A F$ recurrence group, which has more $R A D$, eventually need more permanent pacemaker than the no AF recurrence group.

In the MS group with more than moderate TR, LAVI was still the most discriminative factor. LA enlargement and subsequent LA remodeling and fibrosis is major pathophysiology of AF in the MS group 25. It seems that RA enlargement in the MS group is subsequent change after LA enlargement and tricuspid annulus deformation.

In subgroup analysis of isolated TR, RAD was not a statistically significant factor predicting AF recurrence. Considering that even $L A V I$, which is a known predictor of AF recurrence in lone AF, was not significant indicator of AF recurrence (HR 1.000,95\% Cl [0.983-1.018], p value $<0.969)$ in the isolated TR group, irrelevance of RAD could come from small number of patients with isolated TR $(n=22,13.9 \%)$.

\section{Limitations}

The study has several limitations that need to be addressed. First, this is single center study, so center characteristics could be reflected here. However, a relatively long follow-up duration after surgery (median 7.9 years) is strength of this study. We need external validation moving forward. Second, RAD could be affected by volume status, but there was no measurable value that reflects volume status of patients in 
our data. We should assume that all patients have euvolemic volume status before surgery. Third, because of the limitations of a retrospective study, we could not identify the value of the RA volume index (RAVI). Since RA size has fewer clinical implications than LA size, RAVI was not included in routine protocol. We could include RAVI measurements in a prospective study later. Fourth, there was a limitation in subgroup analysis in patients with isolated TR $(n=22)$ due to the relatively small number of patients. Although we could not reach valuable conclusions in the isolated TR subgroup, it is meaningful that AF recurrence factors are analyzed in end-stage structural heart disease accompanied by significant TR, and this differs from the common-sense view that LA size is most important among echocardiographic parameters in AF recurrence prediction.

\section{Conclusion}

RA enlargement is key predictor of AF recurrence after a modified Cox Maze procedure in patients with significant TR. We can use RA size in clinical risk prediction models to create a risk-benefit estimation of the $\mathrm{CM}$ procedure in patients with significant TR and $\mathrm{AF}$.

\section{Perspectives}

Clinical COMPETENCY in patIENT care and medical knowlege: In patients with significant TR and AF, a $\mathrm{CM}$ procedure at the appropriate time determined by RA diameter could reduce AF recurrence.

TRANSLATIONAL OUTLOOK: Future prospective studies are warranted to determine the AF recurrence prediction power of RAVI measured at euvolemic status in patients with significant TR and AF after TV surgery and CM procedure.

\section{Abbreviations}

Atrial fibrillation $=\mathrm{AF}$

Left atrium volume index $=\mathrm{LAVI}$

Modified Cox maze $=\mathrm{CM}$

Mitral valve stenosis $=\mathrm{MS}$

Right atrium diameter $=\mathrm{RAD}$

tricuspid valve $=\mathrm{TV}$

Tricuspid valve regurgitation $=\mathrm{TR}$

\section{Declarations}




\section{Conflict of Interest Statement}

None of the authors have any conflicts of interest to declare.

\section{Acknowledgments and Funding Sources}

None

\section{References}

1. Lin YJ, Chao TF, Tsao HM, et al. Successful catheter ablation reduces the risk of cardiovascular events in atrial fibrillation patients with CHA2DS2-VASc risk score of 1 and higher. Europace 2013;15(5):676-84. DOI: 10.1093/europace/eus336.

2. Kawaguchi AT, Kosakai Y, Sasako Y, Eishi K, Nakano K, Kawashima Y. Risks and benefits of combined maze procedure for atrial fibrillation associated with organic heart disease. J Am Coll Cardiol 1996;28(4):985-90. DOI: 10.1016/s0735-1097(96)00275-6.

3. Gammie JS, Haddad M, Milford-Beland S, et al. Atrial fibrillation correction surgery: lessons from the Society of Thoracic Surgeons National Cardiac Database. Ann Thorac Surg 2008;85(3):909-14. DOI: 10.1016/j.athoracsur.2007.10.097.

4. Nademanee K, Schwab MC, Kosar EM, et al. Clinical outcomes of catheter substrate ablation for high-risk patients with atrial fibrillation. J Am Coll Cardiol 2008;51(8):843-9. DOI: 10.1016/j.jacc.2007.10.044.

5. Aytemir K, Gurses KM, Yalcin MU, et al. Safety and efficacy outcomes in patients undergoing pulmonary vein isolation with second-generation cryoballoondagger. Europace 2015;17(3):379-87. DOI: 10.1093/europace/euu273.

6. Gerede DM, Candemir B, Vurgun VK, et al. Prediction of recurrence after cryoballoon ablation therapy in patients with paroxysmal atrial fibrillation. Anatol J Cardiol 2015;16(7):482. DOI: 10.5152/AnatolJCardiol.2015.6309.

7. Kim H, Park PW, Sung K et al. Mid-term results of the Cox maze III procedure combined with open mitral commissurotomy for the treatment of rheumatic mitral stenosis. Circ J 2010;74:1332-8.

8. Lang RM, Badano LP, Mor-Avi V, et al. Recommendations for cardiac chamber quantification by echocardiography in adults: an update from the American Society of Echocardiography and the European Association of Cardiovascular Imaging. Eur Heart J Cardiovasc Imaging 2015;16(3):23370. DOI: $10.1093 /$ ehjci/jev014.

9. Kornej J, Hindricks G, Shoemaker MB, et al. The APPLE score: a novel and simple score for the prediction of rhythm outcomes after catheter ablation of atrial fibrillation. Clin Res Cardiol 2015;104(10):871-6. DOI: 10.1007/s00392-015-0856-x.

10. Mujovic N, Marinkovic M, Markovic N, Shantsila A, Lip GY, Potpara TS. Prediction of very late arrhythmia recurrence after radiofrequency catheter ablation of atrial fibrillation: The MB-LATER clinical score. Sci Rep 2017;7:40828. DOI: 10.1038/srep40828. 
11. Chao TF, Ambrose K, Tsao HM, et al. Relationship between the CHADS(2) score and risk of very late recurrences after catheter ablation of paroxysmal atrial fibrillation. Heart Rhythm 2012;9(8):1185-91. DOI: 10.1016/j.hrthm.2012.03.007.

12. Beukema WP, Sie HT, Misier AR, Delnoy PP, Wellens HJ, Elvan A. Predictive factors of sustained sinus rhythm and recurrent atrial fibrillation after a radiofrequency modified Maze procedure. Eur $\mathrm{J}$ Cardiothorac Surg 2008;34(4):771-5. DOI: 10.1016/j.ejcts.2008.07.026.

13. Wu CC, Chang JP, Chen MC, Cheng $\mathrm{Cl}$, Chung WJ. Long-term results of radiofrequency maze procedure for persistent atrial fibrillation with concomitant mitral surgery. $J$ Thorac Dis 2017;9(12):5176-5183. DOI: 10.21037/jtd.2017.11.112.

14. Parwani AS, Morris DA, Blaschke F, et al. Left atrial strain predicts recurrence of atrial arrhythmias after catheter ablation of persistent atrial fibrillation. Open Heart 2017;4(1):e000572. DOI: 10.1136/openhrt-2016-000572.

15. Haïssaguerre $M$, Jaïs $P$, Shah $D C$, et al. Spontaneous initiation of atrial fibrillation by ectopic beats originating in the pulmonary veins. N Engl J Med 1998;339(10):659-66. (In eng). DOI: 10.1056/nejm199809033391003.

16. Calkins H, Hindricks G, Cappato R, et al. 2017 HRS/EHRA/ECAS/APHRS/SOLAECE expert consensus statement on catheter and surgical ablation of atrial fibrillation. Europace 2018;20(1):e1-e160. DOI: 10.1093/europace/eux274.

17. Takagaki M, Yamaguchi $\mathrm{H}$, Ikeda N, et al. Risk Factors for Atrial Fibrillation Recurrence After Cox Maze IV Performed Without Pre-exclusion. Ann Thorac Surg 2020;109(3):771-779. DOI: 10.1016/j.athoracsur.2019.07.016.

18. Moon J, Hong YJ, Shim J, et al. Right atrial anatomical remodeling affects early outcomes of nonvalvular atrial fibrillation after radiofrequency ablation. Circ J 2012;76(4):860-7. DOI: 10.1253/circj.cj-11-1232.

19. Doesch C, Lossnitzer D, Rudic B, et al. Right ventricular and right atrial involvement can predict atrial fibrillation in patients with hypertrophic cardiomyopathy? International journal of medical sciences 2016;13(1):1.

20. Fujimaki $\mathrm{H}$, Mahara K. Impact of atrial fibrillation on tricuspid valve annular morphology in patients with left sided heart valve disease. J Am Coll Cardiol 2017;69 (11 Suppl):1952.

21. Nemoto N, Lesser JR, Pedersen WR, et al. Pathogenic structural heart changes in early tricuspid regurgitation. J Thorac Cardiovasc Surg 2015;150(2):323-30. DOI: 10.1016/j.jtcvs.2015.05.009.

22. Najib MQ, Vinales KL, Vittala SS, Challa S, Lee HR, Chaliki HP. Predictors for the development of severe tricuspid regurgitation with anatomically normal valve in patients with atrial fibrillation. Echocardiography 2012;29(2):140-6. DOI: 10.1111/j.1540-8175.2011.01565.x.

23. Stein JH, Neumann A, Marcus RH. Comparison of estimates of right atrial pressure by physical examination and echocardiography in patients with congestive heart failure and reasons for discrepancies. Am J Cardiol 1997;80(12):1615-8. DOI: 10.1016/s0002-9149(97)00776-5. 
24. John RM, Kumar S. Sinus Node and Atrial Arrhythmias. Circulation 2016;133(19):1892-900. DOI: 10.1161/CIRCULATIONAHA.116.018011.

25. lung $B$, Leenhardt $A$, Extramiana F. Management of atrial fibrillation in patients with rheumatic mitral stenosis. Heart 2018;104(13):1062-1068. DOI: 10.1136/heartjnl-2017-311425.

\section{Table}

Table 1 Baseline Clinical Characteristics Between No AF Recurrence Group and AF Recurrence Group 


\begin{tabular}{|c|c|c|c|}
\hline Variable & No AF recurrence $(n=93)$ & AF recurrence $(n=65)$ & $p$ value \\
\hline \multicolumn{4}{|l|}{ Demographics } \\
\hline Age over 60 & $39(41.9 \%)$ & $38(58.5 \%)$ & 0.041 \\
\hline Sex (female) & $61(65.6 \%)$ & $40(61.5 \%)$ & 0.602 \\
\hline \multicolumn{4}{|c|}{ Cardiovascular risk factors } \\
\hline DM & $12(12.9 \%)$ & $8(12.3 \%)$ & 0.912 \\
\hline HTN & $20(21.5 \%)$ & $14(21.5 \%)$ & 0.996 \\
\hline CKD & $6(6.5 \%)$ & $1(1.5 \%)$ & 0.241 \\
\hline Stroke Hx. & $9(9.7 \%)$ & $8(12.3 \%)$ & 0.600 \\
\hline CAD & $2(2.2 \%)$ & $3(4.6 \%)$ & 0.403 \\
\hline Previous cardiac op & $8(8.6 \%)$ & $5(7.7 \%)$ & 0.838 \\
\hline \multicolumn{4}{|c|}{ Echocardiographic parameters } \\
\hline LVEF (\%) & $55.8 \pm 9.8$ & $56.6 \pm 8.2$ & 0.759 \\
\hline LAD (mm) & $57.3 \pm 9.8$ & $59.1 \pm 10.7$ & 0.266 \\
\hline LAVI $\left(\mathrm{mL} / \mathrm{m}^{2}\right)$ & $98.2 \pm 47.9$ & $120.9 \pm 88.7$ & 0.316 \\
\hline LAE & $92(98.9 \%)$ & $60(92.3 \%)$ & 0.082 \\
\hline RAD (mm) & $51.7 \pm 8.6$ & $58.6 \pm 11.5$ & $<0.001$ \\
\hline RAE & $89(95.7 \%)$ & $64(98.5 \%)$ & 0.649 \\
\hline LVEDD (mm) & $52.1 \pm 9.0$ & $51.9 \pm 9.6$ & 0.843 \\
\hline LVESD (mm) & $34.6 \pm 7.3$ & $34.9 \pm 8.5$ & 0.822 \\
\hline LVMI $\left(\mathrm{g} / \mathrm{m}^{2}\right)$ & $109.1 \pm 42.0$ & $114.6 \pm 48.9$ & 0.755 \\
\hline RVSP (mmHg) & $47.8 \pm 17.5$ & $52.3 \pm 18.4$ & 0.119 \\
\hline$E(m / s e c)$ & $1.64 \pm 0.55$ & $1.53 \pm 0.61$ & 0.244 \\
\hline$e^{\prime}(m / s e c)$ & $0.087 \pm 0.082$ & $0.084 \pm 0.027$ & 0.779 \\
\hline$E / e^{\prime}$ & $22.9 \pm 12.0$ & $20.6 \pm 14.9$ & 0.089 \\
\hline DT (msec) & $438.0 \pm 277.4$ & $440.0 \pm 336.5$ & 0.708 \\
\hline Significant MS & 37 (39.8\%) & $24(36.9 \%)$ & 0.716 \\
\hline Significant MR & $44(47.3 \%)$ & 32 (49.2\%) & 0.812 \\
\hline Significant AS & $11(11.8 \%)$ & $5(7.7 \%)$ & 0.396 \\
\hline
\end{tabular}




\begin{tabular}{|llll|}
\hline Significant AR & $17(18.3 \%)$ & $10(15.4 \%)$ & 0.634 \\
\hline Severe TR & $57(61.3 \%)$ & $48(73.8 \%)$ & 0.168 \\
\hline Isolated TR & $11(11.8 \%)$ & $11(16.9 \%)$ & 0.363 \\
\hline Concomitant procedure & $4(4.3 \%)$ & $7(10.8 \%)$ & \\
\hline CABG & $2(2.2 \%)$ & $6(9.2 \%)$ & 0.202 \\
\hline AVR & $19(20.4 \%)$ & $13(20.0 \%)$ & 0.065 \\
\hline MVR & $55(59.1 \%)$ & $45(69.2 \%)$ & 0.947 \\
\hline DVR & $18(19.4 \%)$ & $12(18.5 \%)$ & 0.195 \\
\hline TVR & $14(15.1 \%)$ & $10(15.4 \%)$ & 0.888 \\
\hline Surgical information & & & 0.955 \\
\hline EuroScore & $5.2 \pm 2.1$ & $5.5 \pm 2.1$ & 0.278 \\
\hline pump time & $149.0[122.0-189.5]$ & $154.0[120.0-208.0]$ & 0.458 \\
\hline ACC time & $115.0[97.0-152.0]$ & $117.0[93.5-155.5]$ & 0.690 \\
\hline
\end{tabular}

Data are presented as mean \pm standard deviation or $\mathrm{n}(\%)$ except pump time and ACC time, pump time and ACC time are presented as median [25 percentile -75 percentiles].

Statistical significance was defined as $p<0.05$ by student $t$-test or Mann Whitney $U$ test (continuous variables) or the Chi-square test or fisher's exact test (categorical variables). The values in bold indicate statistical significance $(p<0.05)$.

$A C C=$ aortic cross clamping; $A R=$ aortic regurgitation; $A S=$ aortic stenosis; $A S D=$ atrial septal defect; $A V R=$ aortic valve replacement; $C A B G=$ coronary artery bypass graft surgery; $C A D=$ coronary artery disease; $C K D$ = chronic kidney disease; $D M=$ diabetes mellitus; $D T$ = deceleration time; DVR(AVR and $M V R)=$ double valve replacement; $E=$ early diastolic mitral inflow velocity; $e^{\prime}=$ early diastolic mitral annular tissue velocity; $H T N$ = hypertension; $L A D$ = left atrium diameter; $L A E$ = left atrium enlargement; $L A V I=$ left atrium volume index; $L V E D D=$ left ventricular end diastolic diameter; $L V E F=$ left ventricular ejection fraction; LVESD = left ventricular end systolic diameter; $L V M I=$ left ventricular mass index; RAE = right atrium enlargement; RAD = right atrium diameter; RVSP = right ventricular systolic pressure; $\mathrm{MR}=$ mitral regurgitation; $\mathrm{MS}$ = mitral stenosis; $\mathrm{MVR}$ = mitral valve replacement; $\mathrm{TR}$ = tricuspid regurgitation; $T V=$ tricuspid valve; TVR = tricuspid valve replacement.

\section{Table 2-1 Clinical Risk Factor for AF Recurrence}




\begin{tabular}{|lllllll|}
\hline Variable & \multicolumn{3}{l}{ Univariable Analysis } & \multicolumn{4}{c|}{ Multivariable Analysis } \\
\cline { 2 - 7 } & HR & $\mathbf{9 5 \%}$ Cl & p value & HR & $95 \%$ Cl & p value \\
\hline Age over 60 & 2.299 & $1.380-3.828$ & 0.001 & 2.299 & $1.380-3.828$ & 0.001 \\
\hline Male Sex & 0.811 & $0.491-1.339$ & 0.412 & & & \\
\hline DM & 1.145 & $0.545-2.404$ & 0.721 & & & \\
\hline HTN & 1.241 & $0.682-2.258$ & 0.480 & & & \\
\hline CKD & 0.310 & $0.043-2.235$ & 0.245 & & & \\
\hline Stroke Hx. & 1.281 & $0.610-2.691$ & 0.514 & & & \\
\hline CAD & 2.239 & $0.696-7.203$ & 0.176 & & & \\
\hline Previous cardiac op & 1.014 & $0.407-2.526$ & 0.976 & & & \\
\hline TV operation method & 1.193 & $0.607-2.346$ & 0.608 & & & \\
\hline
\end{tabular}

$\mathrm{Cl}=$ confidence interval; $\mathrm{HR}=$ hazard ratio; other abbreviations are listed in Table 1.

Table 2-2 Echocardiographic Risk Factor for AF Recurrence

\begin{tabular}{|lllllll|}
\hline Variable & \multicolumn{3}{l}{ Univariable Analysis } & \multicolumn{4}{c|}{ Multivariable Analysis } \\
\cline { 2 - 7 } & HR & $95 \%$ Cl & p value & HR & $95 \%$ Cl & p value \\
\hline LVEF (\%) & 1.003 & $0.976-1.030$ & 0.850 & & & \\
\hline LAVI (ml/m²) & 1.003 & $1.000-1.007$ & 0.033 & 1.003 & $1.000-1.007$ & 0.043 \\
\hline RAD (mm) & 1.044 & $1.023-1.064$ & $<0.001$ & 1.063 & $1.035-1.091$ & $<0.001$ \\
\hline LVEDD (mm) & 0.997 & $0.971-1.022$ & 0.793 & & & \\
\hline RVSP (mmHg) & 1.015 & $1.001-1.030$ & 0.035 & 1.020 & $1.004-1.037$ & 0.017 \\
\hline E/e' & 0.992 & $0.968-1.017$ & 0.532 & & & \\
\hline TR grade & 1.537 & $0.883-2.674$ & 0.129 & & & \\
\hline ASD & 1.692 & $0.771-3.713$ & 0.190 & & & \\
\hline
\end{tabular}

$\mathrm{Cl}=$ confidence interval; $\mathrm{HR}$ = hazard ratio; other abbreviations are listed in Table 1.

Table 3 Right Heart-related Echocardiographic Parameters Comparison Between No AF Recurrence Group and AF Recurrence Group 


\begin{tabular}{|llll|}
\hline Variable & No AF recurrence $(\mathbf{n}=93)$ & AF recurrence $(\mathbf{n}=65)$ & p value \\
\hline TAPSE $(\mathrm{mm})$ & $15.0 \pm 3.6$ & $16.7 \pm 5.1$ & 0.271 \\
\hline TV S' $(\mathrm{m} / \mathrm{s})$ & $0.093 \pm 0.023$ & $0.090 \pm 0.020$ & 0.736 \\
\hline TV annulus $(\mathrm{mm})$ & $40.8 \pm 7.3$ & $42.4 \pm 7.9$ & 0.531 \\
\hline IVC diameter $(\mathrm{mm})$ & $24.5 \pm 4.8$ & $27.3 \pm 5.8$ & 0.008 \\
\hline RV basal diameter $(\mathrm{mm})$ & $47.5 \pm 8.1$ & $51.0 \pm 10.1$ & 0.106 \\
\hline RV mid diameter $(\mathrm{mm})$ & $40.1 \pm 6.1$ & $44.9 \pm 10.4$ & 0.115 \\
\hline RV dilatation & $40(43.0 \%)$ & $37(57.8 \%)$ & 0.068 \\
\hline RV dysfunction & $18(19.4 \%)$ & $11(17.2 \%)$ & 0.831 \\
\hline
\end{tabular}

Data are presented as mean \pm standard deviation or $\mathrm{n}(\%)$.

IVC = inferior vena cava; RV = right ventricle; TAPSE = Tricuspid annular plane systolic excursion; other abbreviations are listed in Table 1.

Statistical significance was defined as $\mathrm{p}<0.05$ by student $\mathrm{t}$-test or Mann Whitney $\mathrm{U}$ test (continuous variables) or Chi-square test or fisher's exact test (categorical variables). The values in bold indicate statistical significance $(p<0.05)$.

Table 4 Comparison of 10 Years Clinical Outcomes Between No AF Recurrence Group and AF Recurrence Group

\begin{tabular}{|c|c|c|c|c|c|c|}
\hline & \multirow{2}{*}{$\begin{array}{l}\text { No AF recurrence (n } \\
=93 \text { ) }\end{array}$} & \multirow{2}{*}{$\begin{array}{l}\text { AF recurrence }(n \\
=65)\end{array}$} & \multicolumn{3}{|c|}{ Univariable Analysis } & \multirow{2}{*}{$\begin{array}{l}\text { Log- } \\
\text { rank } \\
\text { p } \\
\text { value }\end{array}$} \\
\hline & & & HR & $95 \% \mathrm{Cl}$ & $p_{\text {value }}$ & \\
\hline All death & $15(26.7 \%)$ & $13(23.5 \%)$ & 1.106 & $\begin{array}{l}0.525- \\
2.334\end{array}$ & 0.791 & 0.791 \\
\hline TR recurrence & $9(11.0 \%)$ & $8(14.6 \%)$ & 1.277 & $\begin{array}{l}0.492- \\
3.313\end{array}$ & 0.615 & 0.614 \\
\hline $\begin{array}{l}\text { Heart failure } \\
\text { admission }\end{array}$ & $6(7.0 \%)$ & $7(12.3 \%)$ & 1.635 & $\begin{array}{l}0.549- \\
4.870\end{array}$ & 0.377 & 0.373 \\
\hline PPM insertion & $2(3.5 \%)$ & $7(15.1 \%)$ & 4.706 & $\begin{array}{l}0.975- \\
22.708\end{array}$ & 0.054 & 0.034 \\
\hline Ischemic stroke & $0(0.0 \%)$ & $0(0.0 \%)$ & 0.607 & $\begin{array}{l}0.054- \\
6.791\end{array}$ & 0.686 & 0.683 \\
\hline $\begin{array}{l}\text { Hemorrhage } \\
\text { stroke }\end{array}$ & $1(1.6 \%)$ & $3(7.0 \%)$ & 1.899 & $\begin{array}{l}0.425- \\
8.498\end{array}$ & 0.401 & 0.393 \\
\hline
\end{tabular}


Values are $\mathrm{n}(\%)$. Cumulative incidence of events was presented as Kaplan-Meier estimates.

$\mathrm{AF}=$ atrial fibrillation; $\mathrm{Cl}=$ confidence interval; $\mathrm{HR}=$ hazard ratio; $\mathrm{PPM}=$ permanent pacemaker; $\mathrm{TR}=$ tricuspid regurgitation.

\section{Figures}

Samsung Medical Center Institutional Registry 1994.12.15 - 2017.12.28

\section{Patients who did TV operation $(n=421)$}

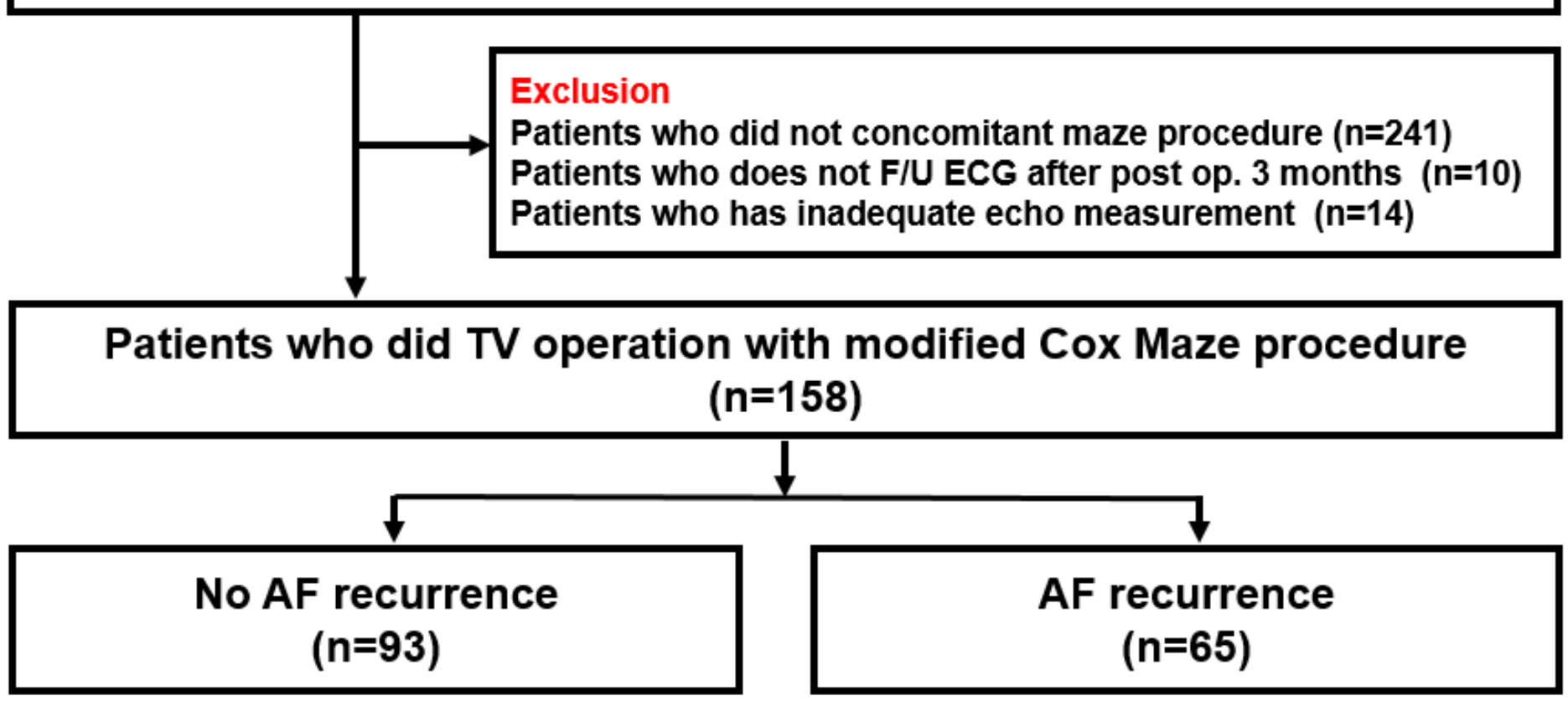

Figure 1

Study flow chart 


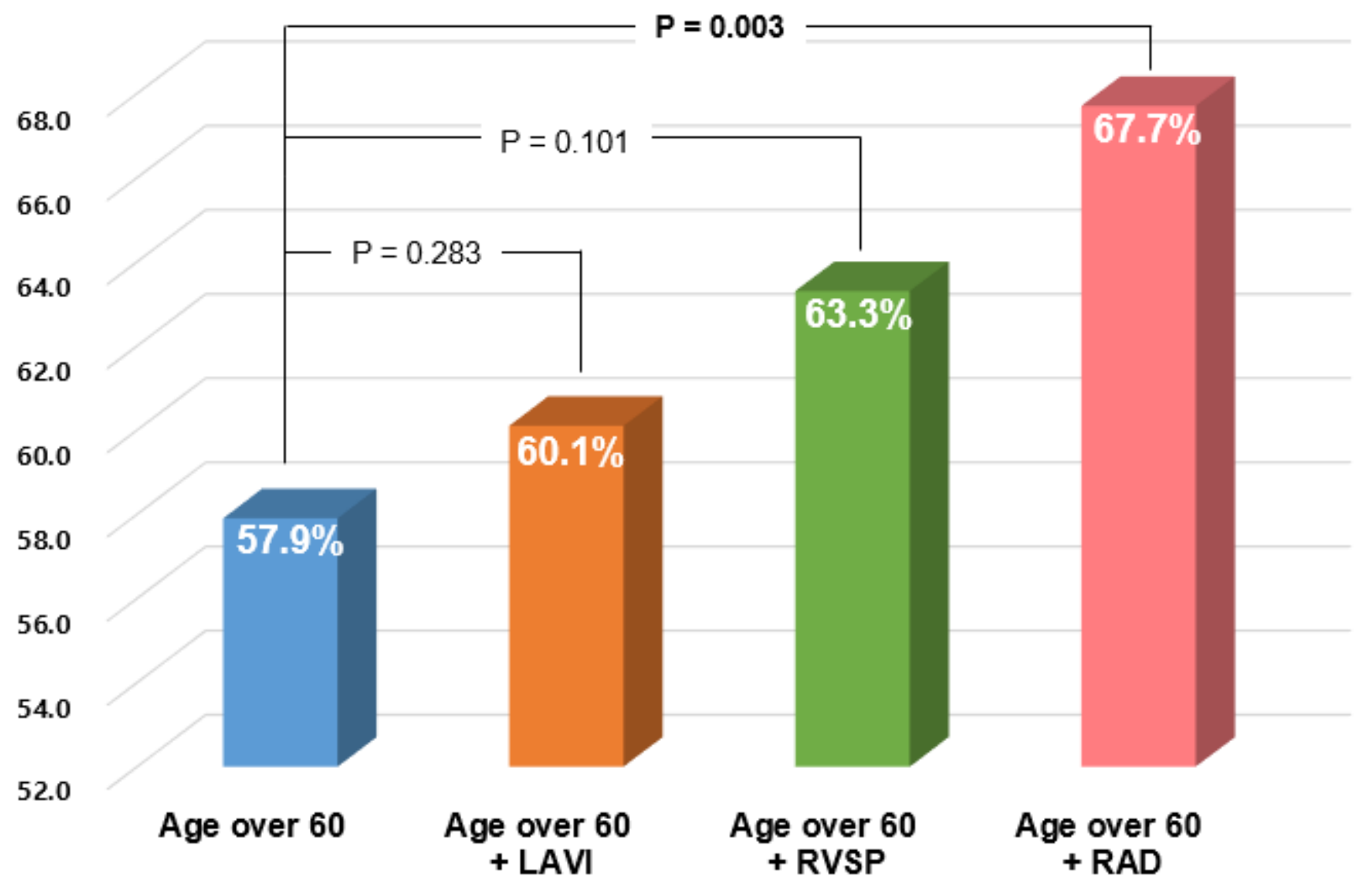

Figure 2

C index comparison for AF recurrence 


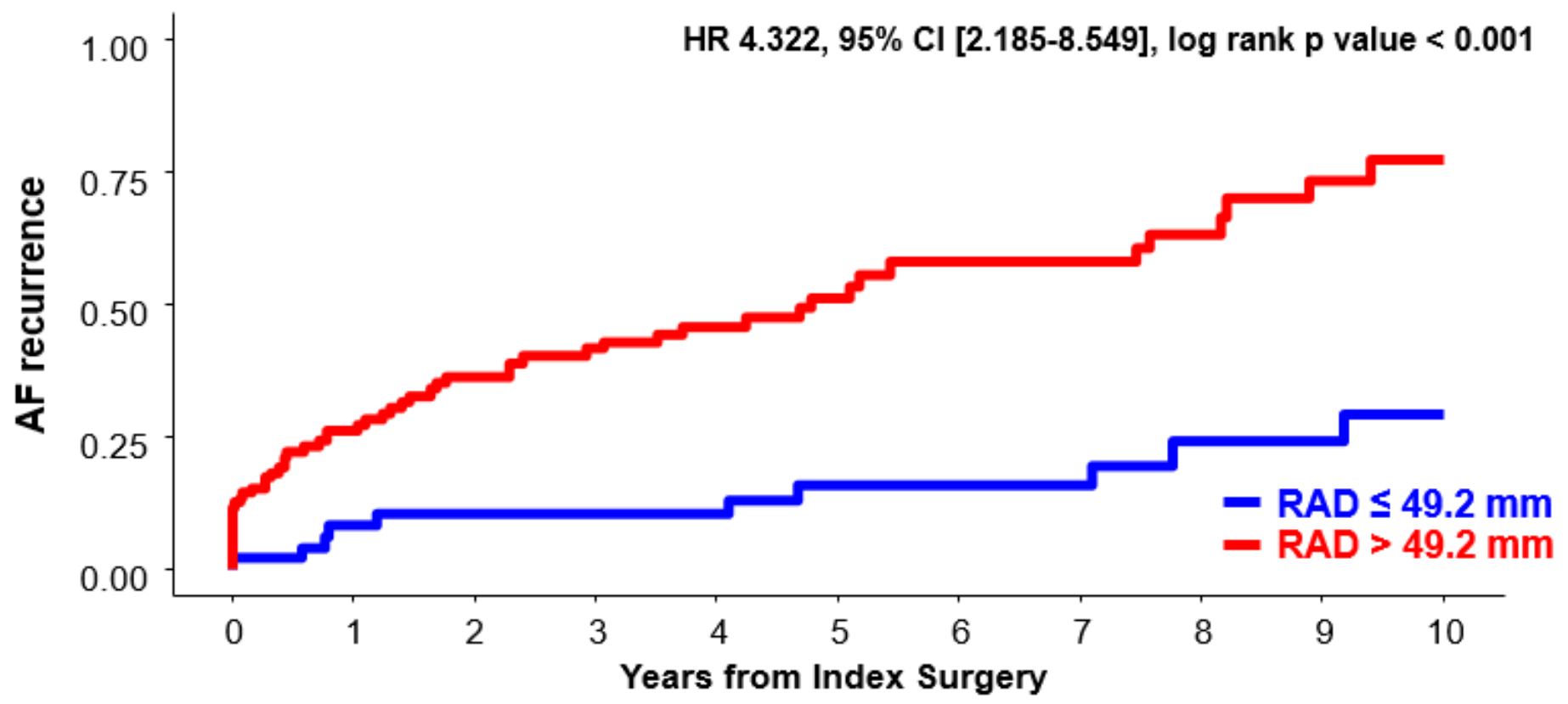

Number at risk

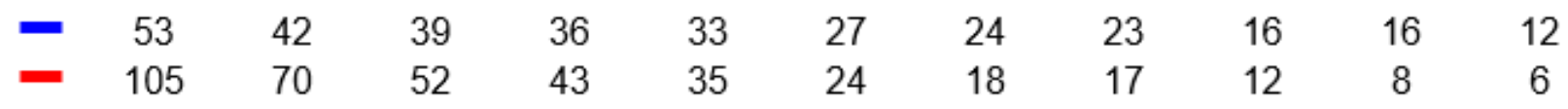

Figure 3

Comparison of AF recurrence according to RA enlargement 


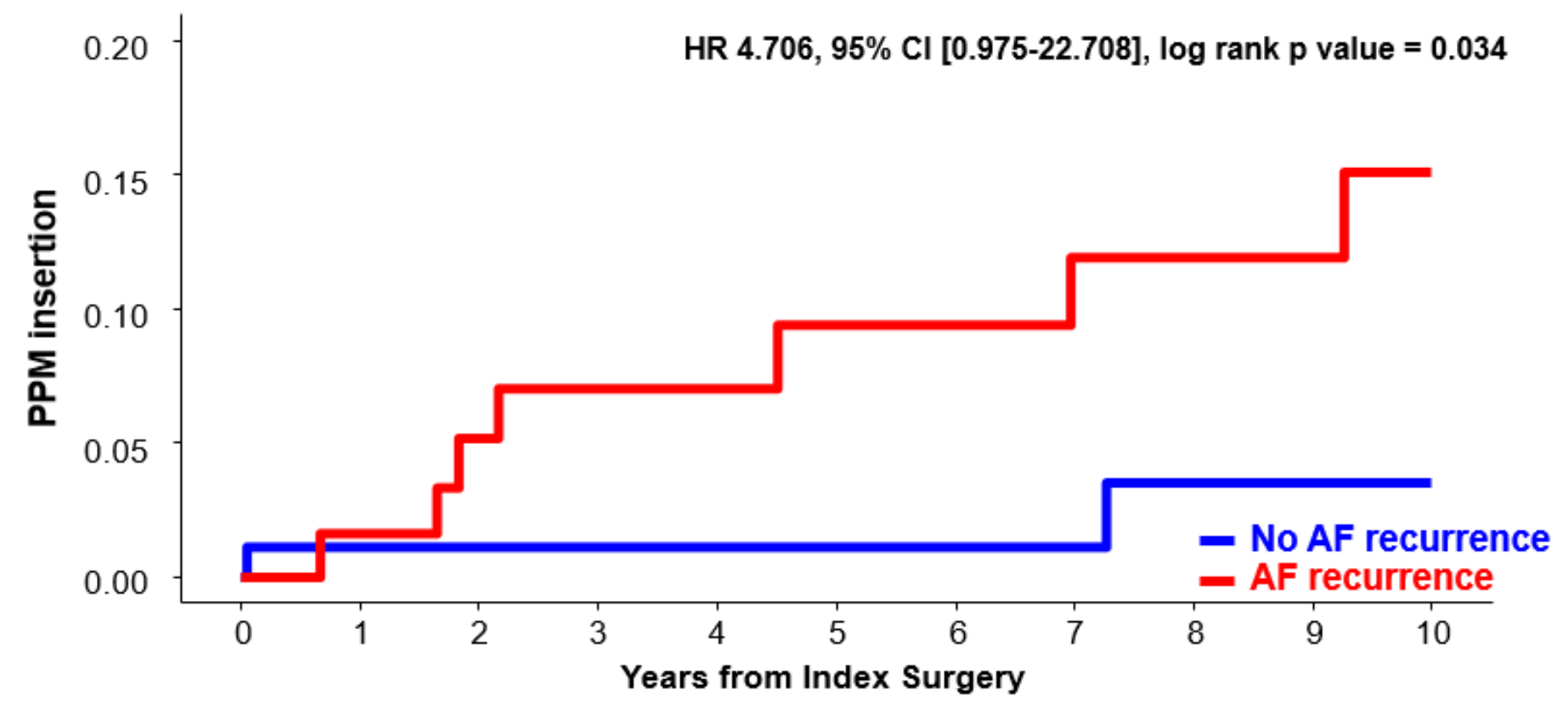

Number at risk

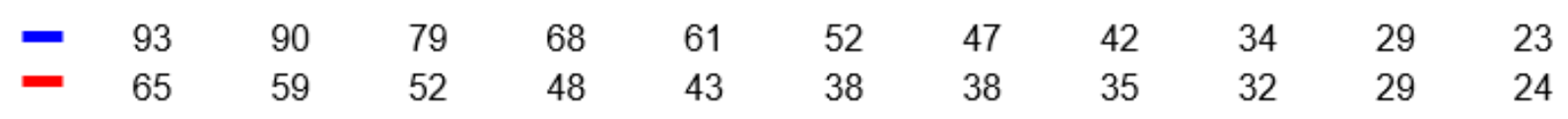

Figure 4

Incidence of PPM insertion according to AF recurrence

\section{Supplementary Files}

This is a list of supplementary files associated with this preprint. Click to download.

- Supplementarytable.docx

- Sup.1.png 